\title{
Performance Evaluation of Downlink WiMAX System in Vicinity of UWB System
}

\author{
Maan A. S. Al-Adwany \\ Department of Computer \& Information Engineering, College of Electronics Engineering \\ University of Mosul \\ Mosul, Iraq \\ maanaladwany@yahoo.com
}

\begin{abstract}
In this paper, we evaluate the performance of WiMAX downlink system in vicinity of $U W B$ system. The study is achieved via simulating a scenario of an office building which utilizes from both WiMAX and UWB appliances. From the simulation results, we found that WiMAX system is largely affected by the $U W B$ interference. However, in order to overcome the interference problem and achieve reasonable BER (Bit Error Rate) of $10^{-4}$, we found that it is very necessary to raise the WiMAX transmitted power in relative to that of $U W B$ interferer. So, the minimum requirements for WiMAX system to overcome UWB interference are stated here in this work.
\end{abstract}

\section{INTRODUCTION}

Ultra Wide Band (UWB) systems will aim to dominate the short-range wireless communications sector facilitating wireless data transmission at rates as high as $110 \mathrm{Mbits} / \mathrm{sec}[1$, 2]. According to the Federal Communication Committee rules, any signal that occupies more than $500 \mathrm{MHz}$ of bandwidth can be considered UWB. Such signals have been allocated in a large frequency band (3.1 to $10.6 \mathrm{GHz}$ ) for unlicensed use [3], with maximum effective isotropic radiated power (EIRP) of $-41.3 \mathrm{dBm} / \mathrm{Mhz}$, in order to avoid interfering with existing narrowband or wideband devices [1]. Although this PSD (Power Spectral Density) mask can effectively suppress UWB interference to most existing systems operating at frequencies lower than $3 \mathrm{GHz}$ (GPRS, GSM 900, GPS, WCDMA and bluetooth), there still exist devices, which operate in the ultrawide spectrum allocated for UWB communications, resulting in mutual interference to both systems.

One of the systems, whose operation could be harmed by UWB interference, is WiMAX system, which is defined by IEEE-802.16 standard. This standard aims to deliver high data rates over large geographical areas to a large number of users [4]. This means that WiMAX will be looking to provide wireless last-mile broadband access with performance comparable to Cable and DSL connections [5]. The operation of WiMAX systems has been restricted in the licensed $2.5 \mathrm{GHz}, 3.5 \mathrm{GHz}$ and license-exempt 5.8GHz. This means that they will share the bands used by UWB systems. So, if UWB technology is to be successful, it is vital for UWB developers to ensure that these devices pose no threat to the operation of WiMAX receivers [6].

However, the effect of UWB interference on WiMAX receivers is studied by many researchers, in [7] (2007), the authors evaluated the impact of UWB transmissions on the performance of a WiMAX receiver. They presented a noncooperative detect and avoid (DAA) procedure to mitigate the UWB interference to WiMAX.

In [8] (2008), the effect of local oscillator (LO) phase noise of an UWB receiver on the detection of a nearby WiMAX receiver was investigated. An expression for the spectrum broadening effect due to the phase noise was derived, and a practical method is presented to estimate the inband interference due to the phase noise interaction with an interfering adjacent channel signal.

In [6] (2009), the coexistence issue between the UWB systems and WiMAX systems has been investigated. The aim was to evaluate the impact that the interference resulting from a realistic UWB hot spot scenario may have on the performance of a WiMAX receiver. The researchers developed a mathematical model through which the performance of the interference power produced by the UWB hot spot can be calculated. Several simulations have been done to both validate the analytical results and calculate the UWB interference.

In [9] (2009), an approach for analyzing the UWB interference effects on the WiMAX downlink has been provided. The maximum permissible interference level, as well as the signal-to-interference level that allows the Ultra Wideband interference effects to be considered as negligible, have been computed by means of analytical, numerical and experimental approaches.

In [10] (2009), the authors experimentally studied the radio coexistence between a WiMAX 802.16e transmission and a WiMedia-defined UWB short-range transmission in a wireless personal area network scenario. The coexistence has been studied evaluating the interference in both directions: First, considering an 802.16 e WiMAX system as a victim of UWB communication and, second, considering the WiMedia- 
defined UWB link as a victim of the WiMAX 802.16e transmission. The experimental results indicated that a victim WiMAX (with $5 \mathrm{MHz}$ bandwidth) exhibit proper operation up to 5 metre transmission in presence of UWB interference if a protection margin of $2.5 \mathrm{~m}$ is met.

In this paper, we study the effect of UWB interference on WiMAX downlink system. In our work we state the minimum requirements for WiMAX to perform well and overcome the UWB interference.

However, this paper is organized as follows: section II presents the WiMAX simulator, also the UWB simulator is introduced in section III. In section IV, we introduce the interference scenario between UWB and WiMAX, while the simulation results are discussed in section V. Finally, the conclusions are drawn in section VI.

\section{WiMAX PHYSICAL LAYER SIMULATOR}

The simulator is based on fixed WiMAX system which differs from mobile WiMAX by its very wide coverage area, in which one base station can cover a small city. While for mobile WiMAX, one base station can cover only about $600 \mathrm{~m}$ cell range.

However, the fixed WiMAX physical layer is based on various communication techniques including coding, interleaving, QPSK modulation, and OFDM transmission. In this section, we elaborate the physical layer of WiMAX downlink system throughout exploring the WiMAX simulator.

Fig. 1 shows the WiMAX simulator which represents a downlink baseband model of the physical layer of a wireless metropolitan area network (WMAN) according to the IEEE 802.16 standard. More specifically, it models the OFDMbased physical layer called Wireless MAN-OFDM, supporting all of the mandatory coding and modulation options.

The tasks performed in the communication system model include $[11,12]$ :

1) Generation of random bit data that models a downlink burst consisting of an integer number of OFDM symbols.

2) Forward Error Correction coding (FEC).

3) Data interleaving.
4) Modulation, using one of the BPSK, QPSK, 16QAM or 64-QAM constellations specified.

5) Orthogonal Frequency Division Multiplexed (OFDM) transmission using 192 sub-carriers, 8 pilots, 256-point FFTs, and a variable cyclic prefix length.

6) A choice of non-fading, flat-fading or selective multipath fading channel.

7) OFDM receiver that includes channel estimation using the inserted preambles.

8) Gain and phase compensation.

9) Data carrier extraction.

10) Hard-decision demodulation followed by deinterleaving and Viterbi decoding.

The model also uses an adaptive-rate control scheme based on SNR estimates at the receiver to vary the data rate dynamically based on the channel conditions.

\section{UWB PhySICAL LAYER SIMULATOR}

Fig. 2 illustrates a simulator for a physical layer of a single user baseband UWB system. The simulator has the following characteristics [13]:

1) Based on IEEE 802.15.3a proposal.

2) Highest mandatory data rate $(200 \mathrm{Mb} / \mathrm{s})$.

3) Rate-5/8 forward error correction coding (convolutional+ puncturing).

4) Data interleaving.

5) QPSK modulation.

6) OFDM transmission: 122 subcarriers, 22 pilots, 128-point FFTs.

7) Frequency hopping and filtering.

8) UWB channel.

9) The receiver including: dehopping and filtering, OFDM receiver, demodulation, deinterleaving, and Viterbi decoding.

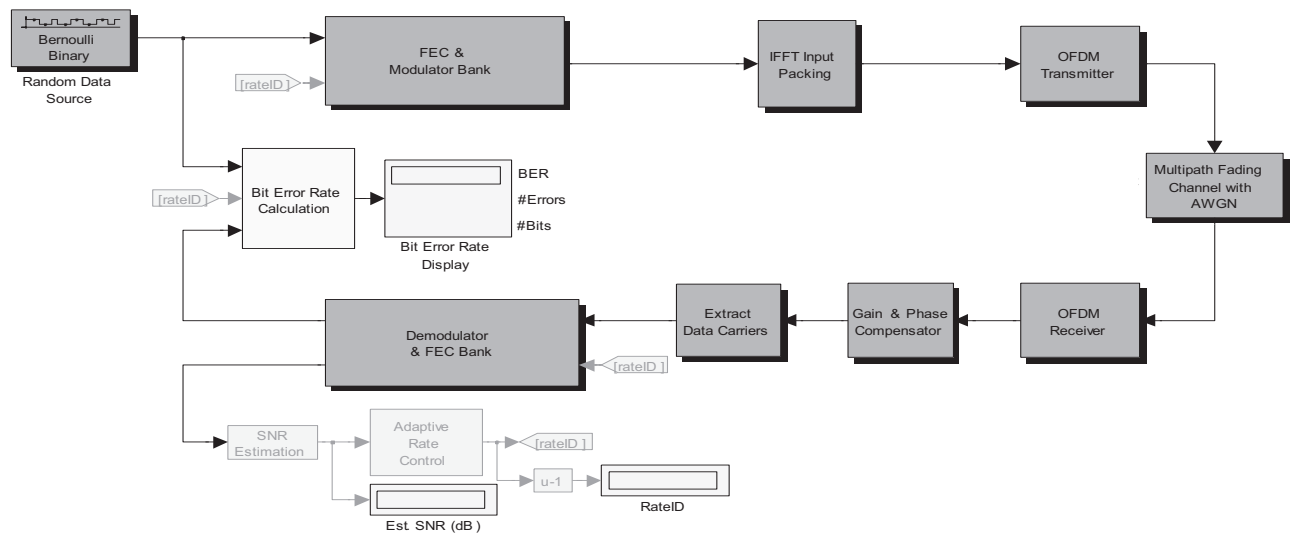

Figure 1. The fixed WiMAX simulator. 


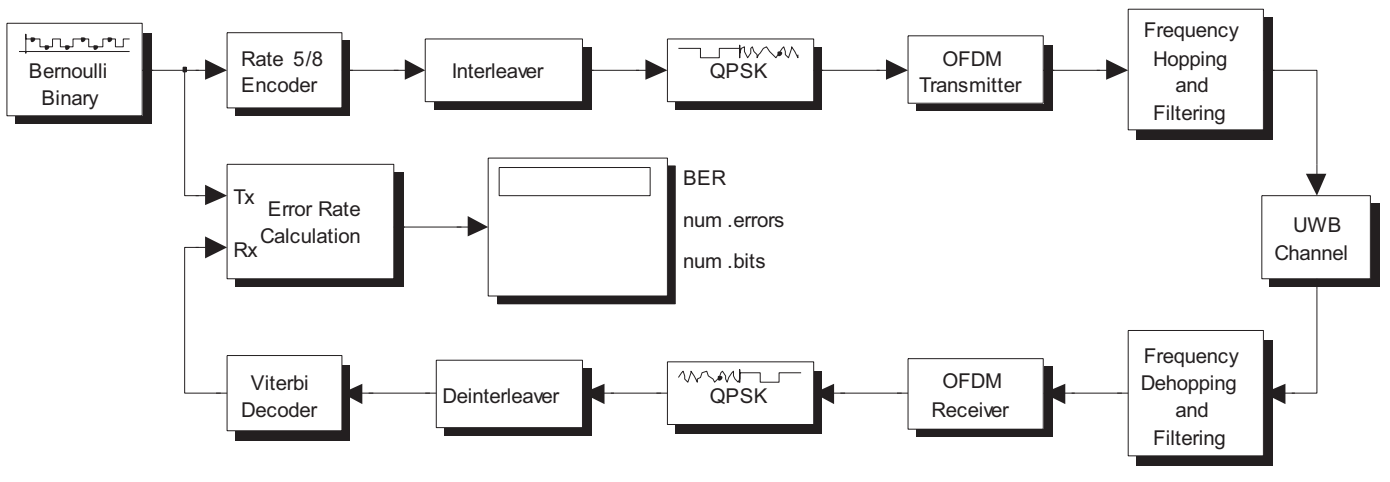

Figure 2. The UWB simulator based on IEEE 802.15.3a proposal.

\section{THE INTERFERENCE SCENARIO}

In this work, we simulate a scenario of UWB system which affects WiMAX receiver fixed on the roof of office building. We suppose that the building utilizes from certain UWB system which is an UWB transmitter which transmits visual data to certain displays in a conferencing environment.

The fixed WiMAX system consists of a base station which transmits data to client receiver fixed on the roof of the building. Also, as stated earlier, we assume that the WiMAX base station has the capability to cover small city with internet and other multimedia services. The distance between the WiMAX base station and the client receiver is denoted as $R$ which is of maximum of $6 \mathrm{~km}$, the city radius. While the distance between the UWB transmitter and the WiMAX client receiver is denoted as $d$.

It is worth to mention that the path loss models used in our simulation are: the vehicular test model for downlink WiMAX, and the free space model for the effect of UWB on WiMAX. Also, for proper operation we assume that the WiMAX base station transmits power above that of UWB by $18 \mathrm{~dB}$.

\section{RESUlTS AND DISCUSSION}

Fig. 3 shows the effect of UWB transmission on the BER (Bit Error Rate) of the WiMAX receiver which uses BPSK modulation. It is obvious that in general and for certain distance $R$, the BER decreases as the distance $d$ between the UWB transmitter and the WiMAX receiver increases. This is because as $d$ increases; the UWB interference decreases which leads to improvement in BER of the WiMAX receiver. Also, for certain $d$, the BER increases as $R$ increases. This can be interpreted as follows: as $R$ increases; the WiMAX signal power decreases due to path loss which in turn reduces the WiMAX signal in relative to UWB interference and hence increasing BER. Furthermore, in order to get a good BER of $10^{-4}$, the distance $d$ must not be less than $8 \mathrm{~m}$ and $16 \mathrm{~m}$ for $R=500 \mathrm{~m}$ and $R=1000 \mathrm{~m}$ respectively. While for maximum reasonable distance $d=25 \mathrm{~m}$, the $10^{-4}$ target BER cannot be

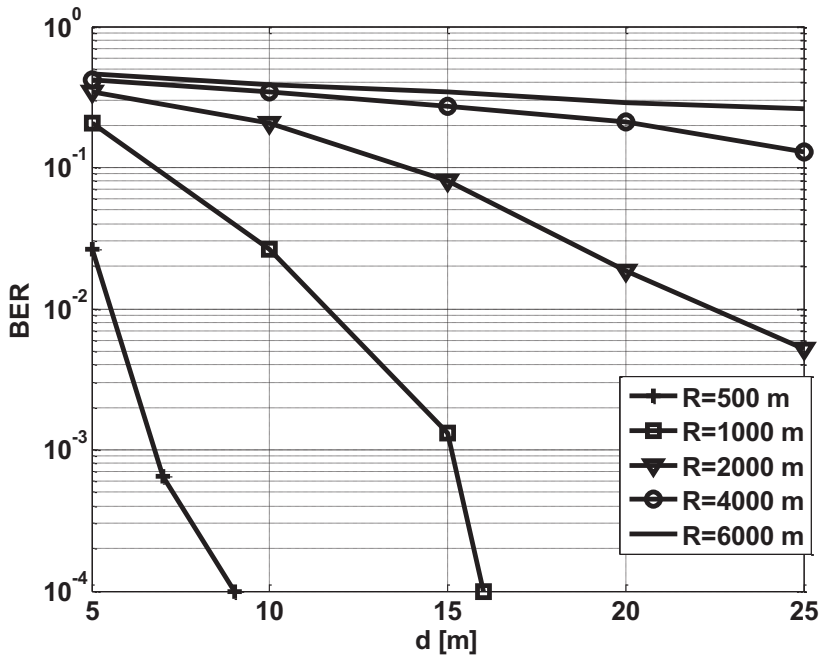

Figure 3. Effect of $d$ on the BER of WiMAX receiver for different values of $R$.

achieved for moderate to large city distances (i.e. $R=2000$ to $6000 \mathrm{~m})$

However, in order to achieve the target BER for moderate to large city distances, we need to raise the WiMAX base station transmitted power in relative to that of UWB to more than $18 \mathrm{~dB}$. For convenient we define $K$ as the relative increment in $\mathrm{dB}$.

Fig. 4 illustrates that for average $d$ of $10 \mathrm{~m}$, and in order to achieve the target BER $\left(10^{-4}\right)$, we need to raise the WiMAX transmitted power above that of UWB by $K=18 \mathrm{~dB}$ for $R=500$, and by $K=24,30,36$, and $38 \mathrm{~dB}$ for distances $R=$ $1000,2000,4000$, and $6000 \mathrm{~m}$ respectively. 


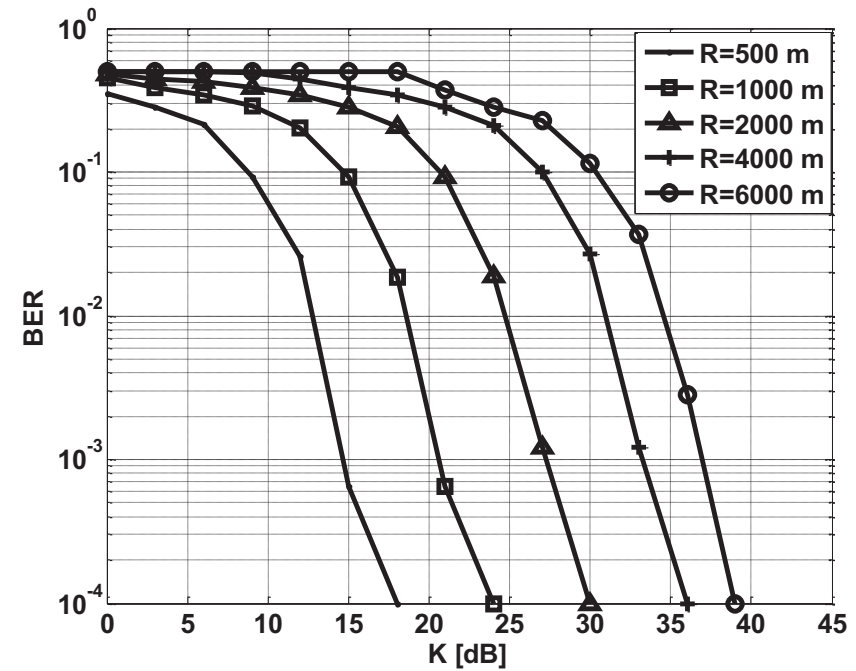

Figure 4. Effect of $K$ on the BER of WiMAX receiver for $d=10 \mathrm{~m}$ and for different values of $R$.

Also, in order to study the possibility of increasing WiMAX spectral efficiency, we use QPSK, 16 QAM, and 64 QAM modulation schemes. It is worth to mention that all the following figures are plotted for average $d$ of $10 \mathrm{~m}$.

Fig. 5 is plotted for $R=500 \mathrm{~m}$, from the figure we can note that as the modulation index goes higher; the required $K$ to achieve the same BER increases. This can be interpreted as follows: in general, in order increase the spectral efficiency; we need to accompany it with increase in signal to interference ratio; and hence increasing $K$. Also, we can note that to achieve the target BER $\left(10^{-4}\right)$ for QPSK, 16 QAM, and 64 QAM, we need to increase $K$ to 22,27 , and $38 \mathrm{~dB}$ respectively.

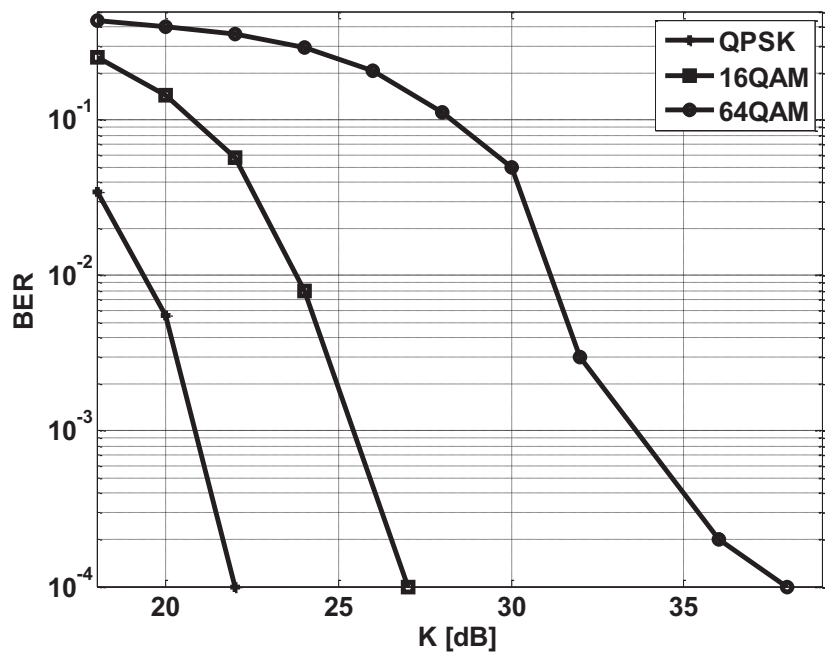

Figure 5. Effect of $K$ on the BER of WiMAX receiver for $R=500 \mathrm{~m}$ and $d=10 \mathrm{~m}$ and for different modulation indexes.
For $R=1000 \mathrm{~m}$, fig. 6 illustrates that to achieve the target BER for QPSK, 16 QAM, and 64 QAM, we need to increase $K$ to 29,33 , and $40.5 \mathrm{~dB}$ respectively. Also, we need to increase $K$ to 34,39 , and $47.5 \mathrm{~dB}$ respectively; for $R=2000 \mathrm{~m}$ as shown in Fig. 7.

Similarly, for $R=4000 \mathrm{~m}$ and in order to achieve the target BER for QPSK, 16 QAM, and 64 QAM, we need to increase $K$ to 40,46 , and $55.5 \mathrm{~dB}$ respectively, as shown in Fig. 8. Finally, to achieve the target BER at the city borders we need to increase $K$ to 44,48 , and $56 \mathrm{~dB}$ respectively, as shown in Fig. 9.

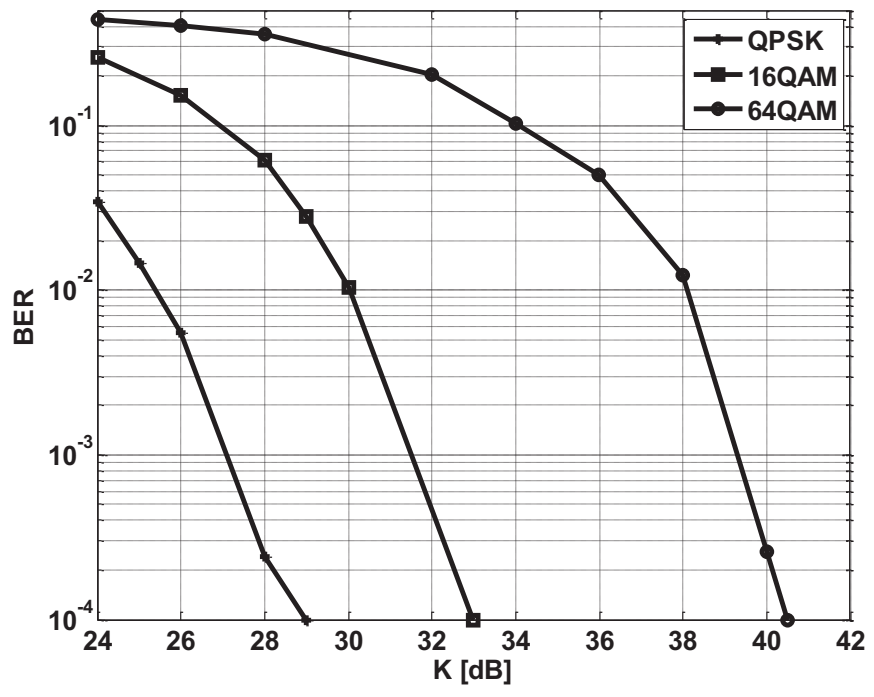

Figure 6. Effect of $K$ on the BER of WiMAX receiver for $R=1000 \mathrm{~m}$ and $d=10 \mathrm{~m}$ and for different modulation indexes.

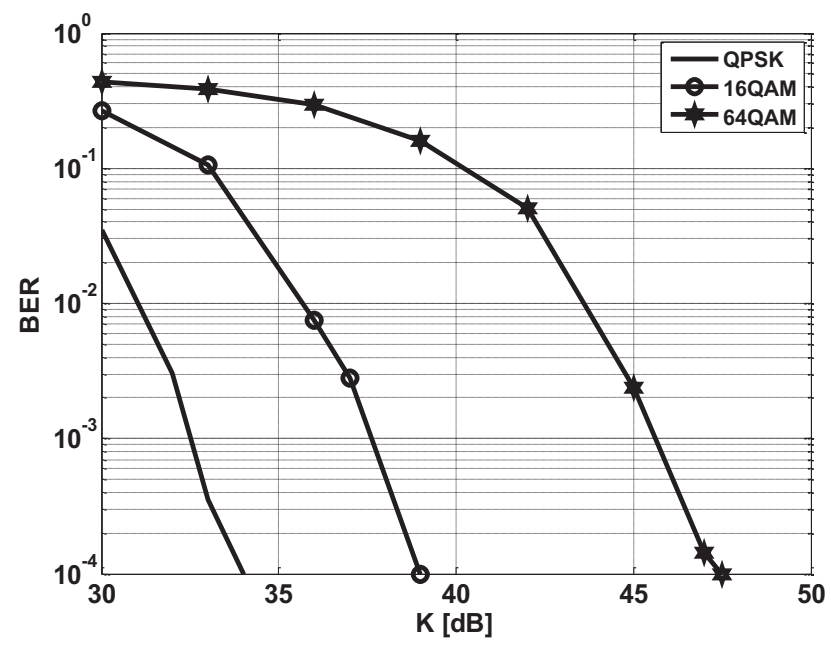

Figure 7. Effect of $K$ on the BER of WiMAX receiver for $R=2000 \mathrm{~m}$ and $d=10 \mathrm{~m}$ and for different modulation indexes. 


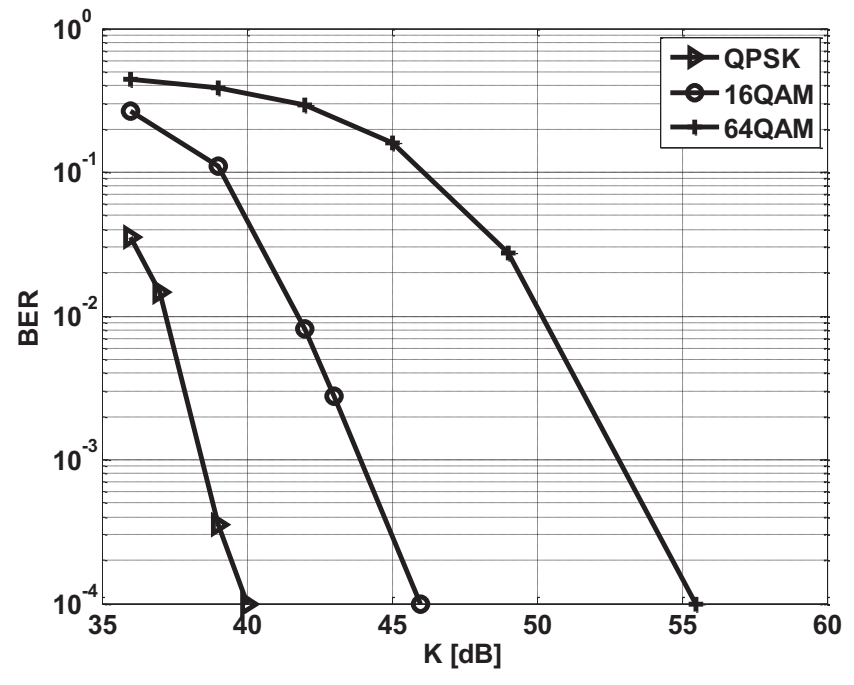

Figure 8. Effect of $K$ on the BER of WiMAX receiver for $R=4000 \mathrm{~m}$ and $d=10 \mathrm{~m}$ and for different modulation indexes.

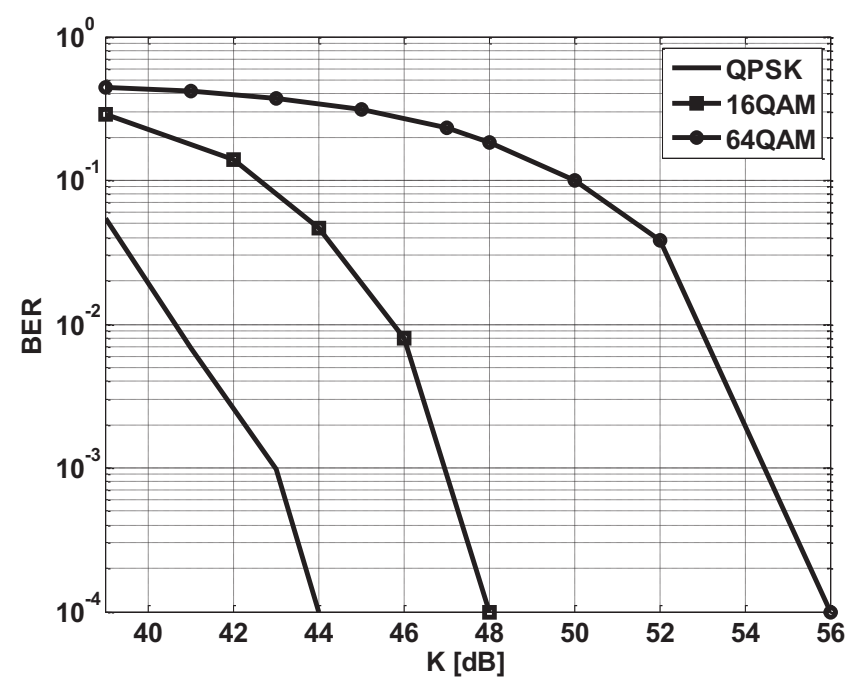

Figure 9. Effect of $K$ on the BER of WiMAX receiver for $R=6000 \mathrm{~m}$ and $d=10 \mathrm{~m}$ and for different modulation indexes.

\section{CONCLUSIONS}

From the simulation results, we can conclude that in order to achieve a reasonable BER at WiMAX receiver and overcome UWB interference, we need to raise the WiMAX transmitted power above that of UWB interferer by considerable amount. The raising amount is essentially depending on the distance between WiMAX receiver and base station, as well as that between WiMAX receiver and UWB transmitter.

\section{ACKNOWLEDGEMENTS}

The author would like to highly thank and appreciate the efforts produced by Ala'a B. Ali, Esra'a H. Najim, and Amina M. Younis, whom assist to succeed this work.

\section{REFERENCES}

[1] R. Giuliano, F. Mazzenga, and D. Porcino, "Use of mitigation techniques to guarantee simultaneous operation of ultra-wide band and third generation cellular radio systems," IST Mobile and Wireless Communications Summit, Lyon, France, June, 2004.

[2] S.S. Mo, A.D. Gelman, "On the power spectral density of UWB signals in IEEE 802.15.3a", Proceeding of Wireless Communications and Networking Conference, Atlanta, USA, vol.2, March 2004, pp. 999-1002.

[3] FCC, "Revision of part 15 of the commissions rules regarding ultrawideband transmission systems", First Report and Order, ET Docket 98-153, FCC 02-8, adopted/released Feb./April 2002.

[4] A. Ghosh, D.R. Wolter, J.G. Andrews, and R. Chen, "Broadband wireless access with WiMax/8O2.16: Current performance benchmarks and future potential", IEEE Communication Mag., vol.43, no.2, Feb. 2005, pp.129-136.

[5] Cisco Systems white paper, "IEEE 802.16 and WiMAX broadband wireless access for everyone", July 2003, available at [www.intel.com/netcomms/ events/ downloads/ ieee80216 wp.pdf].

[6] Andrade, A.G., Parra, V., "Evaluation of UWB Interference at WiMax Systems Based on a Generalized Pulse Waveform", International Conference on Electrical, Communications, and Computers, 2009, CONIELECOMP 2009, pp. 214 - 219.

[7] Durantini, A., Giuliano, R., Mazzenga, F., Valente, D., "Analysis of UWB interference to WiMAX for detect and avoid procedures", International Symposium on Personal, Indoor and Mobile Radio Communications, 2007, PIMRC 2007, pp. 1 - 5 .

[8] Sanghoon Park, Larson, L.E., Milstein, L.B., "Phase noise effects on signal detection for UWB/WiMAX coexistence", Military Communications Conference, MILCOM 2008, pp. 1 - 7 .

[9] Cano, E., Rabbachin, A., Fortuny, J., "Performance analysis of WiMAX systems in the presence of MB-OFDM UWB interference", 6th International Symposium on Wireless Communication Systems, ISWCS 2009, pp. $264-268$.

[10] Perez, J., Beltran, M., Morant, M., Llorente, R., Cavallin, L., "Protection margins for joint operation of WiMAX 802.16e and WiMedia-defined UWB radio in personal area networks", International Conference on Ultra-Wideband, ICUWB 2009, pp. $723-727$

[11] Available at [www.mathworks.com], last accessed date: 4/1/2009.

[12] IEEE 802.16-2004 Standard Specification, available at [http://ieee802.org/16/pubs/80216-2004.html], last accessed date: 20/1/2009.

[13] Martin Clark and Mike Mulligan, "UWB fixed-point model (multiband OFDM)", 2004, available at [http//www.mathworks.com/matlabcentral/fileexchange/45], last accessed date: 14/11/2008. 